\title{
Effects of self-prepared hypertonic nasal saline irrigation in allergic rhinitis: A randomized controlled trial
}

\author{
Kittiyaporn Sansila, ${ }^{1}$ Peem Eiamprapai, ${ }^{2}$ Ratree Sawangjit ${ }^{3,4}$
}

\begin{abstract}
Background: Nasal saline irrigation has been reported to be effective as an adjunctive therapy for allergic rhinitis (AR), but concerns about adverse events, supply problems, and high costs have limited its widespread clinical use. Aqueous $1.8 \%$ sodium chloride solution prepared by patients using drinking water (1.8\% self-prepared hypertonic nasal saline irrigation; $1.8 \%$ SPHNSI) could solve some of these problems, but its clinical efficacy and safety need to be determined.
\end{abstract}

Objective: We aimed to compare the efficacy and safety of $1.8 \%$ SPHNSI and $0.9 \%$ commercial isotonic nasal saline irrigation (0.9\% CINSI) in patients with AR.

Methods: A randomised, single-blinded, placebo-controlled trial was performed as a pilot study. Seventy-eight patients with AR were included. Each patient was randomised to nasal irrigation with $80 \mathrm{~mL}$ of either $1.8 \%$ SPHNSI or $0.9 \%$ INSI twice-daily for 4 weeks. Randomised codes were generated using a computer and a block of 4 procedure. The primary outcome was improvement of quality of life scores in Thai patients with allergic rhinoconjunctivitis (Rcq-36). Secondary outcomes were clinical symptoms using total nasal symptom scores (TNSS) and adverse events. All outcomes were assessed by blinded assessors at baseline, week 2 , and week 4 .

Results: At week 4, nasal irrigation with $1.8 \%$ SPHNSI had significantly improved the Rcq-36 score (54\% versus 50\%; $p<0.032)$ and congestion symptom score $(96 \%$ versus $84 \% ; p<0.018)$ compared to nasal irrigation with $0.9 \%$ CINSI. Adverse events were comparable for both groups at week 4.

Conclusion: This pilot study indicates that regular use of $1.8 \%$ SPHNSI in AR patients for 4 weeks is safe and has superior efficacy to $0.9 \%$ CINSI for alleviating congestion and improving quality of life scores.

Key words: Self-prepared hypertonic nasal saline irrigation (SPHNSI), allergic rhinitis (AR), total nasal symptom scores (TNSS), Rcq-36, randomised controlled trial

\section{From:}

Department of Clinical Pharmacy, Faculty of Pharmacy, Mahasarakham University, Khamriang, Kantarawichai, Maha Sarakham, Thailand.

2 Department of Otolaryngology, Faculty of Medicine, Mahasarakham University, Talat, Mueang, Maha Sarakham, Thailand.

Clinical Trials and Evidence-Based Syntheses Research Unit (CTEBs RU), Department of Clinical Pharmacy, Faculty of Pharmacy, Mahasarakham University, Khamriang, Kantarawichai, Maha Sarakham, Thailand.

${ }^{4}$ School of Pharmacy, Monash University Malaysia,

Jalan Lagoon Selatan, Bandar Sunway, Selangor, Malaysia

\section{Corresponding author:}

Ratree Sawangjit

Clinical Trials and Evidence-Based Syntheses Research Unit (CTEBs RU), Department of Clinical Pharmacy, Faculty of Pharmacy,

Mahasarakham University, Kantarawichai, Maha Sarakham, 44150

Thailand

E-mail: ratree.m@msu.ac.th

\section{Introduction}

Allergic rhinitis (AR) is one of the most common chronic diseases worldwide including Thailand, with an estimated prevalence of $10 \%$ to $45 \% .^{1-5}$ Although AR is not a life-threatening condition, its symptoms include sneezing, rhinorrhoea, nasal itching, and congestion, and it has a profound impact on an individual's physical, social, and emotional well-being a well as quality of life (QoL) of patients. ${ }^{6}$ Treatment options for AR may be non-pharmacologic such as avoidance of the allergen, or pharmacologic therapies such as oral/intranasal non-sedating antihistamines or decongestants, or intranasal corticosteroids. ${ }^{7-8}$ In addition, nasal irrigation with hypertonic or isotonic saline has been used as an adjunctive therapy in several countries including Thailand. ${ }^{9-12}$ With nasal saline irrigation, the composition and concentration of the sodium chloride solution can vary. ${ }^{12}$ Current evidence suggests that 
hypertonic nasal saline irrigation (HNSI) with concentrations of $2-6 \%(\mathrm{w} / \mathrm{v})$ sodium chloride is superior to isotonic nasal saline irrigation (INSI) for relief of nasal symptoms. ${ }^{10,12-14} \mathrm{~A}$ possible explanation for this is that the hypertonic solution reduces mucosal oedema due to osmotic pressure-induced water transport through the mucosal epithelial membrane, thereby reducing nasal congestion and improving mucociliary clearance. ${ }^{10}$ However, adverse effects are also reported to increase with increasing concentrations of sodium chloride. ${ }^{12-15}$

High costs and supply problems with commercial isotonic nasal saline or hypertonic nasal saline can limit the clinical use of nasal saline irrigation in patients with AR. ${ }^{16}$ To resolve this problem, various formulations of home-made or self-prepared nasal saline irrigations (SPNSI) have been proposed. ${ }^{16}$ Conventional methods of preparing SPNSI are time-consuming and inconvenient, however, requiring patients to mix sodium chloride with boiled water and leave it to cool before use. In this study, we investigated a simpler method in which an aqueous $1.8 \%(\mathrm{w} / \mathrm{v})$ sodium chloride solution was prepared using drinking water without boiling ( $1.8 \%$ sodium chloride nasal saline irrigation; $1.8 \%$ SPHNSI). This work builds upon the findings of a previous phase I study conducted with healthy Thai volunteers, ${ }^{17}$ which showed that participant satisfaction with the safety domain of $1.8 \%$ SPHNSI was superior to that with $0.9 \%$ self-prepared isotonic nasal saline irrigation $(0.9 \%$ SPINSI) and no different from $0.9 \%$ commercial isotonic nasal saline irrigation (CINSI) ${ }^{17}$ Unlike the previous study, however, we compared not just the safety but also the efficacy of $1.8 \%$ SPHNSI treatment with $0.9 \%$ CINSI treatment in AR patients.

\section{Methods}

A randomised single-blinded, active-controlled, parallelgroup trial was conducted at three community hospitals (Sahatsakhan Hospital, Kalasin Hospital, and Suddhavej Hospital) in Thailand to determine the efficacy and safety of $1.8 \%$ SPHNSI compared with a controlled commercial isotonic nasal saline irrigation ( $0.9 \%$ CINSI). The study was conducted in compliance with the principles of good clinical practice (GCP) and in accordance with the Declaration of Helsinki, and the study protocol was approved by the Ethics Committee of Mahasarakham University, Thailand. Written informed consent was obtained from all AR participants before enrolment. The study is reported in accordance with CONSORT recommendations for randomised controlled trials, ${ }^{18-19}$ and the study protocol is registered at www.clinicaltrials.in.th (\#T CTR20150923001). Seventy-eight patients with allergic rhinitis who gave their informed consent were screened using inclusion-exclusion criteria. The inclusion criteria for this study were as follows: (1) patients with age $>18$ years, (2) patients who had a history of allergic cause and one or more of the following symptoms: nasal congestion, runny nose, itchy nose, or sneezing, (3) patients who presented with one or more of the following symptoms: nasal congestion, runny nose, itchy nose, or sneezing more than 4 days/week and more than 4 weeks/year diagnosed by a physician using history and physical examination. Patients with a history of food or drug allergy, abnormal vital signs following good clinical practice of the hospital (blood pressure $>140 / 90 \mathrm{mmHg}$, pulse rate $>110$ beat $/ \mathrm{min}$, body temperature $>37.8^{\circ} \mathrm{C}$ ), abnormal nasal ciliary function, and rhinosinusitis or upper respiratory tract infection based on patient history and physical examination were excluded.

\section{Interventions and blinding process}

All patients were randomly assigned to receive either the treatment product or the control product. The treatment product was a self-prepared hypertonic nasal saline irrigation with a concentration of $1.8 \%(1.8 \%$ SPHNSI). All patients in the treatment group were instructed to prepare fresh $1.8 \%$ SPHNSI by mixing $5.94 \mathrm{~g}$ of prepacked sodium chloride (pharmaceutical grade) with $330 \mathrm{~mL}$ of drinking water (DW) obtained from sealed bottles. The control product was $0.9 \%$ sodium chloride (isotonic) commercial saline solution (0.9\% CINSI). The mean $\mathrm{pH}$ values of $1.8 \%$ SPNSI and $0.9 \%$ CINSI were 7.81 \pm 0.36 and $5.8 \pm 0.5$, respectively. The set for each product consisted of a similar sealed opaque package, which was opened only in the intervention room. Patients were not blinded to the difference in interventions. However, the physicians or outcome assessors were blinded by separating the rooms for product distribution, irrigation process, and outcome measurement. In addition, patients were asked not to disclose their treatment or show their products to the physicians. All physicians received standard training before the trial started, and each patient was assessed by the same physician throughout the study duration.

The nasal irrigation technique was explained by the same instructor at each setting. Each participant was advised to use a $20 \mathrm{~mL}$ disposable syringe for consecutive irrigation with 80 $\mathrm{mL}$ of saline solution in each nostril two times/day at home for 4 weeks. A brief demonstration of proficiency with the nasal irrigation technique was required before patient departure.

During the study period, each participant was allowed to continue using previous medications for control of their rhinitis symptoms, such as intranasal corticosteroids, without changing their dosage regimen. For oral antihistamines and decongestants, participants were allowed to use these only when required.

\section{Outcomes and measurement tools}

All outcomes were assessed by blinded physicians at baseline (before nasal irrigation) on day 0 and after nasal irrigation on week 2 and week 4 (the end of the study). The primary outcome was improvement of QoL scores using a questionnaire specific for Thai allergic rhinoconjunctivitis patients (Rcq-36), which was validated for Thai people. ${ }^{20}$ The Rcq-36 was composed of 36 items divided into seven domains including rhinitis symptoms (RS), eye symptoms (ES), other symptoms (OS), physical functioning $(\mathrm{PF})$, role limitations $(\mathrm{RL})$, sleep problems (SP), social functioning (SF), emotions (E), and overall health $(\mathrm{OH})$, with a rank of 1 equating to no impairment at all and 5 indicating maximum impairment. Secondary outcomes were clinical symptom scores using a total nasal symptom score (TNSS) assessment. Nasal symptoms recordedin this study were nasal congestion, nasal itching, nasal discharge, and sneezing. All symptoms were graded on a 4-point scale from 0 (no symptoms) to 3 (severe symptoms that are bothersome and interfere with daily activities or disturb sleep). ${ }^{10,21}$ 
Therefore, higher scores in both assessments indicate a more severe presentation of chronic AR. All patients were asked to recall the problems mentioned in the questionnaire or symptoms during the previous 2 weeks. All sets of questions were answered verbally by patients. Adverse events were also assessed as secondary outcomes.

\section{Statistical analysis}

The sample size calculated to be necessary for detecting a difference in the QoL between the intervention and control groups was 172 patients. The sample size was calculated based on a result from a previous study, ${ }^{10}$ with type 1 error of 0.05 and $80 \%$ power to detect the difference of four scores in the QoL using an Rcq-36 questionnaire between a $0.9 \%$ self-prepared nasal saline irrigation and a $0.9 \%$ commercial nasal saline irrigation. This calculation factored in a possible withdrawal rate of $10 \%$. Because of limitations in time and funding, approximately $45 \%$ of the desired number of patients were enrolled in this pilot study.
All analyses used the intention-to-treat approach. Descriptive statistics were used to describe patient characteristics such as severity of disease, adverse events, comorbidity, and number of concomitant medicines. The average score of QoL or symptom score at the end of the study between groups was compared using the Man-Whitney $U$ test or independent t-test depending on data distribution. The average scores within a group at all measurement times were analysed using repeated one-way ANOVA or Friedman test depending on data distribution. A chi-squared test was used to analyse the proportion or binary outcome. All $p$-values were two sided, and $p$-values less than 0.05 were considered statistically significant.

\section{Results}

Between September 2015 and March 2016, 80 patients were screened for enrollment, 78 of whom were randomised. Thirty -five patients were assigned to the $1.8 \%$ SPHNSI group, and 43 patients were assigned to the $0.9 \%$ CINSI group. Two patients in the $1.8 \%$ SPHNSI group and four patients in the $0.9 \%$ CINSI group were lost to follow-up or dropped out during the study period (Figure 1).

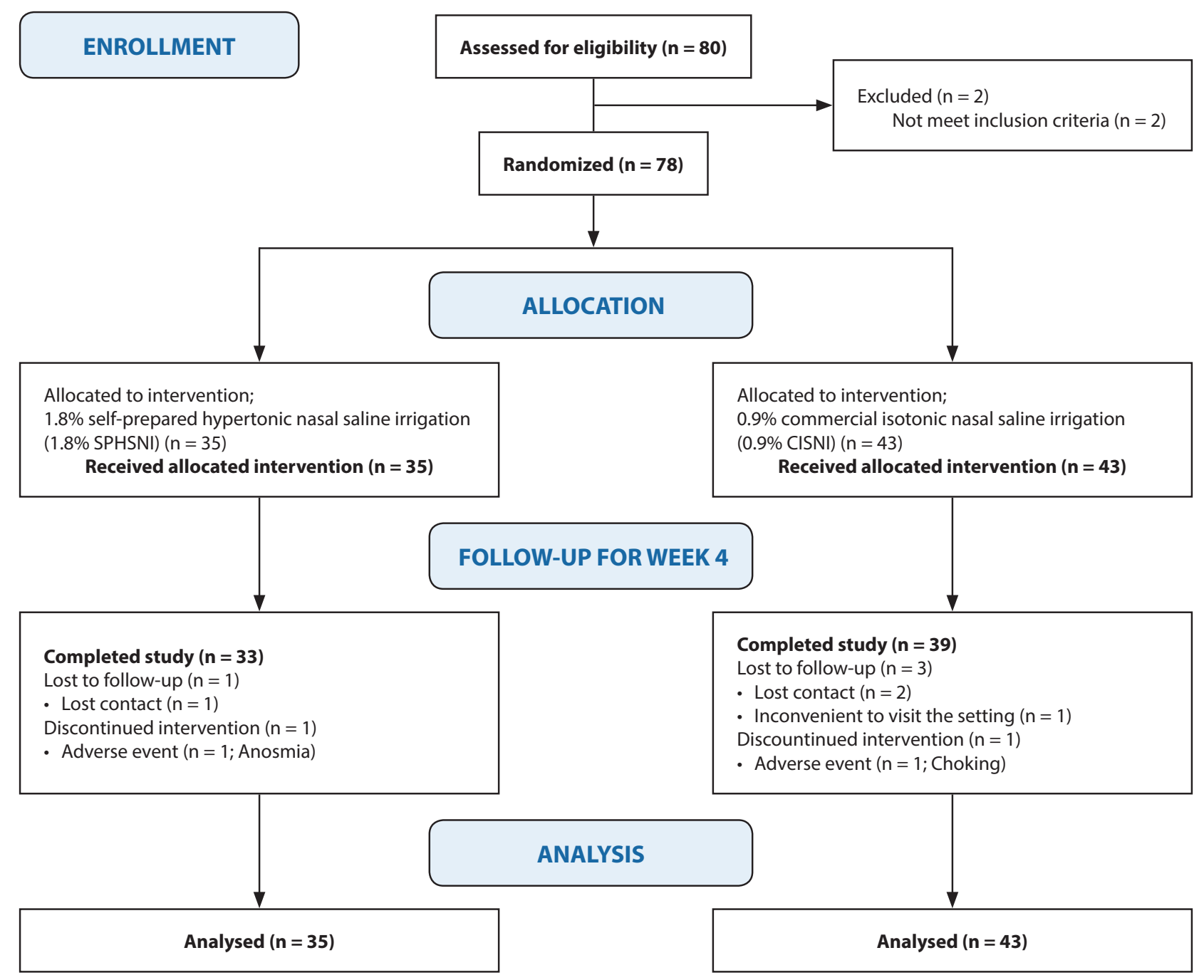

Figure 1. Flow of patients through trial 
Table 1. Baseline characteristics

\begin{tabular}{|c|c|c|c|}
\hline Parameters & $\begin{array}{l}1.8 \% \text { SPHNSI } \\
\quad(n=35)\end{array}$ & $\begin{array}{l}0.9 \% \text { CINSI } \\
(n=43)\end{array}$ & P-value \\
\hline Age (years); mean + sd & $38.37+11.47$ & $35.61+11.07$ & 0.249 \\
\hline Female; n (\%) & $26(74.71)$ & $28(65.12)$ & 0.383 \\
\hline \multicolumn{3}{|c|}{ Range of total nasal scores; $\mathrm{n}(\%)$} & 0.772 \\
\hline - Scores 1-4 & $4(11.43)$ & $7(16.28)$ & \\
\hline - Scores 5-8 & $22(62.86)$ & $24(55.81)$ & \\
\hline - Scores 9-12 & $9(25.71)$ & $12(27.91)$ & \\
\hline \multicolumn{4}{|c|}{ Frequency and severity of AR; $n(\%)$} \\
\hline - Persistent & $35(100.00)$ & $43(100.00)$ & $>0.999$ \\
\hline - Moderate-severe & $35(100.00)$ & $43(100.00)$ & $>0.999$ \\
\hline \multicolumn{4}{|l|}{ Comorbidity; n (\%) } \\
\hline - Asthma & $2(5.71 \%)$ & $1(2.32)$ & 0.585 \\
\hline - COPD & $0(0)$ & $1(2.32)$ & $>0.999$ \\
\hline \multicolumn{4}{|c|}{ Concomitant medicines ${ }^{\epsilon} ; \mathrm{n}(\%)$} \\
\hline $\begin{array}{l}\text { - Oral } \\
\text { anti-histamine }\end{array}$ & $18(51.42 \%)$ & $25(58.14 \%)$ & 0.553 \\
\hline - Oral decongestant & $18(51.42 \%)$ & $20(46.51 \%)$ & 0.280 \\
\hline $\begin{array}{l}\text { - Intranasal } \\
\text { corticosteroid }\end{array}$ & $5(14.29 \%)$ & $6(13.95 \%)$ & 0.666 \\
\hline $\begin{array}{l}\text { Total nasal scores } \\
\text { (TNSS); mean + sd }\end{array}$ & $7.40+2.23$ & $7.35+2.59$ & 0.881 \\
\hline - Sneezing & $1.69+0.83$ & $1.70+0.80$ & 0.872 \\
\hline - Congestion & $2.06+0.68$ & $1.98+0.86$ & 0.795 \\
\hline - Itching & $1.89+0.72$ & $1.70+0.89$ & 0.343 \\
\hline - Rhinorrhea & $1.77+0.65$ & $1.95+0.79$ & 0.313 \\
\hline $\begin{array}{l}\text { Quality of life (QoL); } \\
\text { mean + sd }\end{array}$ & $105.80+28.56$ & $112.30+24.52$ & 0.374 \\
\hline $\begin{array}{l}\text { - Rhinitis symptoms } \\
\text { (RS) }\end{array}$ & $14.31+3.14$ & $15.16+3.06$ & 0.111 \\
\hline - Eye symptoms (ES) & $11.77+4.70$ & $13.12+3.63$ & 0.161 \\
\hline $\begin{array}{l}\text { - Other symptoms } \\
\text { (OS) }\end{array}$ & $23.49+7.85$ & $23.93+7.49$ & 0.888 \\
\hline $\begin{array}{l}\text { - Physical } \\
\text { functioning }(\mathrm{PF})\end{array}$ & $9.00+3.60$ & $9.33+3.21$ & 0.750 \\
\hline $\begin{array}{l}\text { - Role limitations } \\
\text { (RL) }\end{array}$ & $7.74+2.80$ & $8.88+2.82$ & 0.060 \\
\hline $\begin{array}{l}\text { - Sleep problems } \\
\text { (SP) }\end{array}$ & $9.29+3.19$ & $10.09+2.83$ & 0.323 \\
\hline $\begin{array}{l}\text { - Social functioning } \\
\text { (SF) }\end{array}$ & $9.54+3.81$ & $10.02+2.99$ & 0.784 \\
\hline - Emotions (E) & $16.74+5.12$ & $17.79+3.85$ & 0.446 \\
\hline $\begin{array}{l}\text { - Overall health } \\
(\mathrm{OH})\end{array}$ & $3.91+0.78$ & $3.98+0.64$ & 0.772 \\
\hline
\end{tabular}

n: number of participants; AR: allergic rhinitis; ${ }^{\epsilon}$ : some participants were taking more than one concomitant medicine; sd: standard deviation.

\section{Demographic data}

Seventy-eight patients including 54 females (69\%) and $24(31 \%)$ males with an average age of $36.85 \pm 11.26$ years (range 18-77 years) were enrolled and included in the intention-to-treat analysis (ITT analysis) (Figure 1). Baseline characteristics of patients are shown in Table 1. Both groups had similar baseline characteristics. All of them were classified as persistent AR with moderate to severe symptoms. Most of them had TNSS ranging from 5-12 (46 of 78 patients; $59 \%$ ) and used antihistamine as a concomitant medicine (43 of 78 patients; $55 \%)$. The average total clinical symptom scores for treatment and control groups were $7.20 \pm 2.49$ and $7.02 \pm 2.82$, respectively. The summary of QoL scores for treatment and control groups were $105.00 \pm 28.56$ and $112.30 \pm 24.52$, respectively (Table 1).

\section{Effectiveness of nasal saline irrigation}

The results of the pre- and post-nasal irrigation at week 2 and week 4 in terms of QoL and clinical symptoms (TNSS) are shown in Table 2. At week 4, patients treated with $1.8 \%$ SPHNSI had a statistically significant improvement in the total QoL score compared to those treated with $0.9 \%$ CINSI ( $p$-value $=0.032$; percent improvement of QoL scores in $1.8 \%$ SPHNSI and $0.9 \%$ CINSI were $54 \%$ and $50 \%$, respectively). When evaluating each QoL domain at week 4, patients treated with $1.8 \%$ SPHNSI had a statistically significant improvement in role limitations (RL) and emotion (E) domains compared to those treated with $0.9 \%$ CINSI (Table 2). In addition, the nasal congestion symptom scores significantly decreased in the $1.8 \%$ SPHNSI group compared to the $0.9 \%$ CINSI group at week 4 ( $p$-value $=0.018$; percent improvement of congestion scores in $1.8 \%$ SPHNSI and $0.9 \%$ CINSI were $96 \%$ and $84 \%$, respectively). Moving on to within-group comparisons, the QoL and TNSS scores in AR patients using 1.8\% SPNSI or $0.9 \%$ CINSI both significantly decreased in all domains at week 2 and week 4 following initiation of nasal saline irrigation (Table 2).

\section{Safety of nasal saline irrigation}

Reports of adverse events are shown in Table 3. Adverse events in patients treated with $1.8 \%$ bSPHNSI (54\%) were significantly higher than those in patients treated with $0.9 \%$ CINSI (28\%) at week 2. However, the number of these adverse events decreased and were seen equally in both groups at week 4 (Table 3). Only one patient in each group dropped out due to adverse events (Figure 1). The most common adverse event was nasal irritation with mild severity (no need for additional treatment). In addition, the number of adverse events decreased when nasal saline irrigation was used continually for 4 weeks (Table 3). 


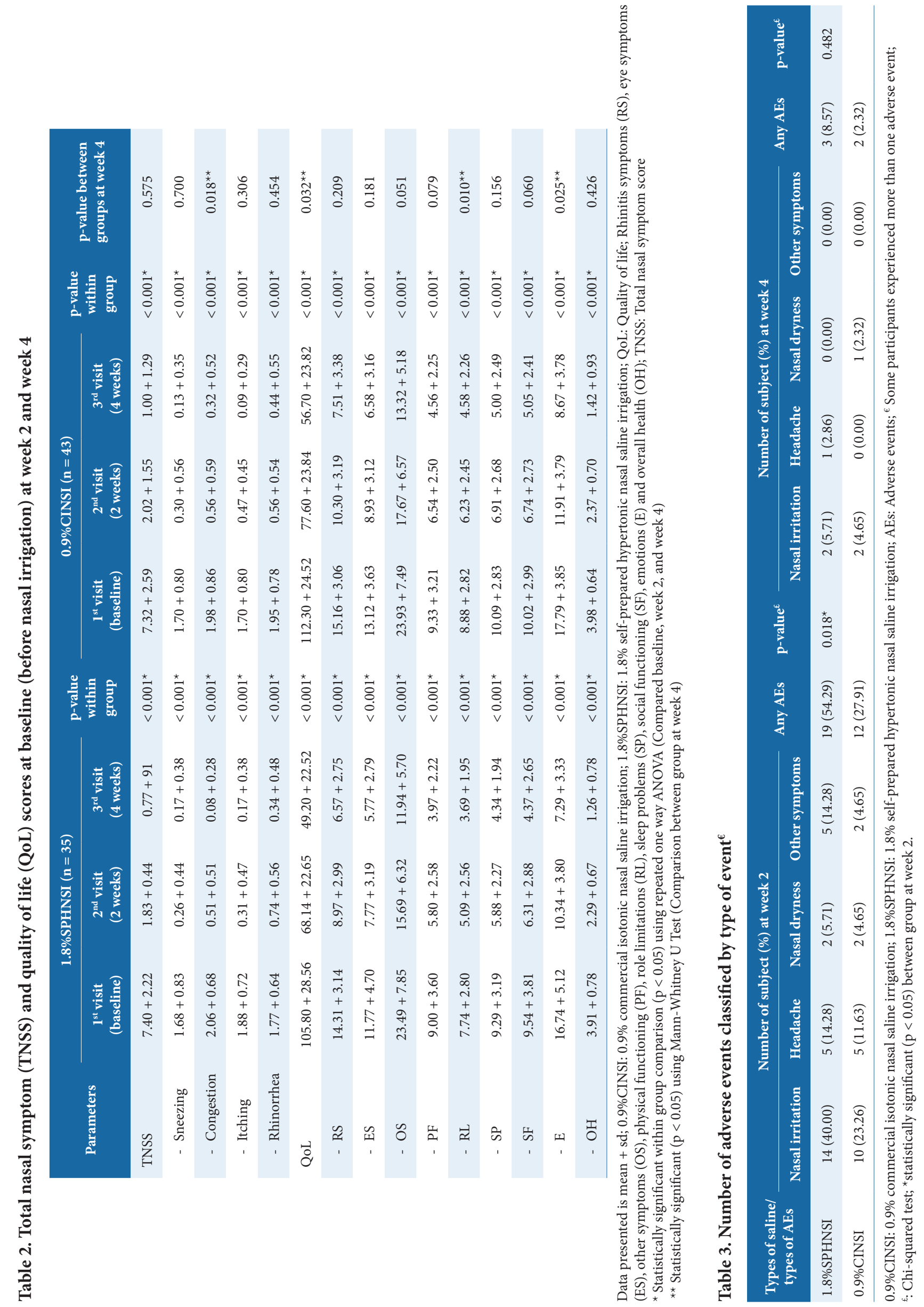




\section{Discussion}

Nasal irrigation using different tonicities of sodium chloride solution have been used in various studies. ${ }^{10-15}$ This pilot study was performed to compare the efficacy and safety of nasal irrigation using hypertonic (1.8\% SPHNSI) or isotonic nasal saline irrigation $(0.9 \%$ CINSI). Our results indicate that both clinical symptoms (congestion symptoms) and the QoL of patients with AR are significantly improved when nasal irrigation is performed for 4 weeks with 1.8\% SPHNSI instead of $0.9 \%$ CINSI. In addition, both $1.8 \%$ SPHNSI and $0.9 \%$ CINSI can improve all clinical symptoms evaluated and QoL domains at week 2 and week 4 compared to baseline results. Adverse events were similar in both groups at week 4 .

Our findings are consistent with previous studies ${ }^{9-15}$ showing that nasal saline irrigation is an effective adjunctive treatment for AR, at least in the short-term. Since patients in this study were allowed to concurrently use their previous medications with nasal irrigation, we suggest using hypertonic saline or isotonic saline irrigation as an add-on or adjunctive therapy with standard medications.

With regard to ethical issues, this study allowed each participant to continue using their previous medications including intranasal corticosteroids, oral antihistamines, and/or decongestants for control of rhinitis symptoms during the study period. Concurrent use of different medications during the study period was considered a confounding factor. However, we planned to decrease the effects of this confounder by stratified randomisation using TNSS scores, which related to the medication(s) used. The study results show that types and proportions of medication used were similar in both groups at baseline, implying that the effect of this confounder was balanced between groups.

The superior effectiveness of $1.8 \%$ SPHNSI to $0.9 \%$ CINSI in this study may be attributable to the tonicity and $\mathrm{pH}$ of this saline solution. Previous studies suggested that hypertonic saline solution leads to a greater improvement in mucociliary activity and nasal symptoms compared to isotonic solution. ${ }^{22-25}$ Both mucociliary activity and nasal symptoms were also better when buffered nasal saline irrigation ( $\mathrm{pH} 7.2-8.4)$ was used instead of unbuffered nasal saline irrigation $\left(\mathrm{pH}\right.$ 6.2-6.4). ${ }^{24-26}$ According to previous studies, ${ }^{23-27}$ a hypertonic saline solution (1.8\% SPHNSI) with mild alkalinity ( $\mathrm{pH} \sim 8$ ) should have superior efficacy to isotonic saline $(0.9 \%$ CINSI) with mild acidity ( $\mathrm{pH} \sim 6$ ).

Generally, saline solutions prepared using distilled water will always be acidic unless a buffer is added. ${ }^{27}$ The mild acidity of $0.9 \%$ CINSI in our study was consistent with previous findings ${ }^{27}$ because this solution was prepared from distilled water without adding buffer. The $1.8 \%$ SPHNSI, by contrast, was mildly alkaline. The difference in $\mathrm{pH}$ of each saline formulation was consistent with previous studies indicating that use of different types of water leads to different $\mathrm{pH}$ values in the prepared saline solutions. ${ }^{17}$ Preparing $1.8 \%$ SPHNSI using sealed bottles of drinking water without adding any buffer resulted in a mildly alkaline property that may have enhanced the efficacy of the saline solution.

The primary strength of our work is that it was the first randomised, controlled trial to investigate the efficacy and safety of $1.8 \%$ SPHNSI, a hypertonic nasal saline solution prepared by a simple process, for allergic rhinitis. Our study was performed and reported following good clinical practice guidelines and adheres to CONSORT guidelines. ${ }^{17-18}$ Moreover, both the physicians (outcome assessors) and pharmacists (product distributors) were blinded. These methods can decrease selection and detection biases, and help ensure high quality and internal validity of findings. In addition, this study included AR patients with symptoms of varying severity who were using a variety of medications, so our findings are likely to be generalisable to the broader AR patient population. As stated previously, a randomisation code was used to stratify the TNSS score by range, related to AR severity and concurrent medication(s), to balance this confounding factor between the $1.8 \%$ SPNSI and 0.9\% CINSI groups. Furthermore, this study minimised potential bias by ensuring each patient was examined by the same investigator for the duration of the study.

A few limitations in our study should be highlighted. First, due to differences in product characteristics (self-prepared nasal saline vs. commercial nasal saline), patients were not blinded. This limitation may produce performance bias in patients who know which products they used. Second, we used an accurate mass of pharmaceutical grade salt instead of an approximate mass of cooking salt. This was done to improve the accuracy of results, but does not represent what is likely to occur in patients' homes. In low-middle income countries such as Thailand, patients cannot accurately weigh salt at home, so these conditions are not generalisable to real life. Additional studies to mimic the real situation in homes should, therefore, be performed, so that an actual recommendation can be made to patients. Third, this was only a preliminary study with a small sample size and short time of follow-up, thus differences detected had with low power. Significant differences were detected in clinical symptoms and QoL between groups, but further study with an appropriate sample size and longer term of follow-up would help confirm these findings. Also, since other sinonasal pathologies such as acute sinusitis, acute $\mathrm{AR}$, and chronic sinusitis were not included, generalisations to these patients cannot be made. Finally, whilst we followed AAO NHSF guideline criteria for diagnosing allergic rhinitis (patient history and physical examination), ${ }^{8}$ allergy testing was not performed and we cannot exclude the possibility that some participants were suffering from non-allergic rhinitis. AAO NHSF guidelines ${ }^{8}$ are widespread in use, however, so this limitation does not affect the generalisability of our findings to clinical practise in most hospitals, where allergy testing is not performed.

From a clinical viewpoint, 'clinical significance' can be as important as 'statistical significance. The minimal clinically important difference (MCID) illustrates the relationship between outcome measures and the patient's perception of change, by calculating the smallest change in a given outcome that is meaningful to patients. For the measurement of TNSS (on a 12-point scale) in allergic rhinitis, MCID threshold values of 0.23 units on the 4-point scales of each domain ${ }^{28}$ and 3.6 points from 12-point scales ${ }^{29}$ have been reported. For the measurement of quality of life in allergic rhinitis, an MCID threshold of 5.9 units from total scores ${ }^{28}$ has been reported. Considering MCID of the TNSS score, $1.8 \%$ SPHNSI showed 
a clinically significant improvement in congestion score compared to $0.9 \%$ CINSI at week 4 . In addition, both $1.8 \%$ SPHNSI and $0.9 \%$ CINSI at weeks 2 and 4 showed a clinically significant improvement on both 12-point and 4-point scales of each domain compared to baseline. Also, 1.8\% SPHNSI showed a clinically significant improvement in total quality of life scores compared to $0.9 \%$ CINSI at week 4 . Clinically significant improvement was also found at weeks 2 and 4 after nasal irrigation with $1.8 \%$ SPHNSI or $0.9 \%$ CINSI compared to baseline. Importantly, these results show that improvements in congestion and total quality of life scores were significantly superior at 4 weeks, both statistically and clinically, in patients treated with $1.8 \%$ SPHNSI instead of $0.9 \%$ CINSI.

In low-middle income countries such as Thailand and Lao PDR, the clinical use of nasal saline irrigation is limited due to supply problems and the high cost of commercial saline. Data presented here indicates that $1.8 \%$ SPHNSI could solve these problems for AR patients. We found 1.8\% SPHNSI to be safe and to provide superior benefits compared to $0.9 \%$ CINSI. The $1.8 \%$ SPHNSI was also easy to prepare, and cheaper than CINSI.

\section{Conclusions}

This pilot study indicates that regular use of $1.8 \%$ SPHNSI in AR patients for 4 weeks is safe and has superior efficacy to 0.9\% CINSI for alleviating congestion and improving QoL scores. Our findings, therefore, support the use of saline irrigation as an adjunctive treatment to standard medications. Further studies are now needed to determine safety and efficacy over a longer term. Further studies are also warranted to determine the safety and efficacy of nasal irrigation in patients with other sinonasal pathologies.

\section{Acknowledgements}

The authors thank Asst. Prof. Dr. Methin Phadungkit and Asst. Prof. Dr. Bunleu Sungthong for useful suggestions on quality control of the pre-mix sachets of salt, Dr. Sitta Jirakulsomchok and Dr. Poomchai Romsaithong for data collection, and all participants for their help. They also thank Dr. Tim Cushnie for language-editing assistance, and Dr. Chanuttha Ploylearmsang for her kindness in statistical consulting.

\section{Conflicts of interest}

All authors confirm that there are no known conflicts of interest associated with this publication from the past 3 years.

\section{Funding}

This study received partial funding from Mahasarakham University Faculty of Pharmacy.

\section{Author contributions}

Kittiyaporn Sansila, Peem Eiamprapai, and Ratree Sawangjit were responsible for the study concept and design. Kittiyaporn Sansila and Peem Eiamprapai contributed to data collection. All authors acquired, analysed, or interpreted the data. Ratree Sawangjit drafted the manuscript. All authors critically revised the manuscript for important intellectual content. Ratree Sawangjit supervised the study and is the corresponding author.

\section{References}

1. Bunnag C, Jareoncharsri P, Tantilipidorn P, Vichyanond P, Pawankar R. Epidemiology and current status of allergic rhinitis and asthma in Thailand. Asian Pac J Allergy Immunol. 2009;27(1):79-86.

2. Beasley R, Keil U, von Mutius E, Pearce N. Worldwide variation in prevalence of symptoms of asthma, allergic rhinoconjunctivitis, and atopic eczema: ISAAC. The International Study of Asthma and Allergies in Childhood (ISAAC) Steering Committee. Lancet. 1998; 351(9111):1225-32.

3. Uthaisangsook S. Prevalence of asthma, rhinitis, and eczema in the university population of Phitsanulok, Thailand. Asian Pac J Allergy Immunol. 2007;25(2-3):127-32.

4. Teeratakulpisarn J, Wiangnon S, Kosalaraksa P, Heng S. Surveying the prevalence of asthma, allergic rhinitis and eczema in school-children in Khon Kaen, Northeastern Thailand using the ISAAC questionnaire: phase III. Asian Pac J Allergy Immunol. 2004;22(4):175-81.

5. Trakultivakorn M, Sangsupawanich P, Vichyanond P. Time trends of the prevalence of asthma, rhinitis and eczema in Thai children-ISAAC (International Study of Asthma and Allergies in Childhood) Phase Three. J Asthma. 2007;44(8):609-11.

6. Juniper E, Guyalt G, Dolovich J. Assessment of quality of life in adolescents with allergic rhinoconjunctivitis: development and testing of a questionnaire for clinical trial. Allergy Clin Immunol. 1994;93(2):413-23.

7. Brožek JL, Bousquet J, Agache I, Agarwal A, Bachert C, Bosnic-Anticevich $\mathrm{S}$, et al. Allergic rhinitis and its impact on asthma (ARIA) guidelines-2016 revision. J Allergy Clin Immunol. 2017;140(4):950-8.

8. Seidman MD, Gurgel RK, Lin SY, Schwartz SR, Baroody FM, Bonner JR, et al. Clinical practice guideline: allergic rhinitis executive summary. Otolaryngol Head Neck Surg. 2015;152(2):197-206.

9. Lipworth B, Newton J, Ram B, Small I, Schwarze J. An algorithm recommendation for the pharmacological management of allergic rhinitis in the UK: a consensus statement from an expert panel. NPJ Prim Care Respir Med. 2017;27(1):3.

10. Satdhabudha A, Poachanukoon O. Efficacy of buffered hypertonic saline nasal irrigation in children with symptomatic allergic rhinitis. Int J Pediatr Otorhinolaryngol. 2012;76:583-8.

11. Hermelingmeier KE, Weber RK, Hellmich M, Heubach CP, Mosges R. Nasal irrigation as an adjunctive treatment in allergic rhinitis: A systematic review and meta-analysis. Am J Rhinol Allergy. 2012;26(5): e119-25.

12. Head K, Snidvongs K, Glew S, Scadding G, Schilder AG, Philpott C, et al. Saline irrigation for allergic rhinitis. Cochrane Database Syst Rev 2018;6: CD012597.

13. Kanjanawasee D, Seresirikachorn K, Chitsuthipakorn W, Snidvongs K. Hypertonic saline versus isotonic saline nasal irrigation: systematic review and meta-analysis. Am J Rhinol Allergy. 2018;32(4):269-79.

14. Malizia V, Fasola S, Ferrante G, Cilluffo G, Montalbano L, Landi M, et al. Efficacy of buffered hypertonic saline nasal irrigation for nasal symptoms in children with seasonal allergic rhinitis: a randomized controlled trial. Int Arch Allergy Immunol. 2017;174(2):97-103.

15. Marchisio P, Varricchio A, Baggi E, Bianchini S, Capasso ME, Torretta S, et al. Hypertonic saline is more effective than normal saline in seasonal allergic rhinitis in children. Int J Immunopathol Pharmacol. 2012;25(3): 721-30.

16. Lilic N, Waldvogel-Thurlow S, Douglas RG. Physical characteristics of commercial and home-made nasal lavage solutions. J Laryngol Otol. 2014;128 Suppl 1:S40-3.

17. Sansila K, Kaenphukhieo T, Khochuaiklang P, Eiamprapai P, Sungthong B, Sawangjit R. Properties and safety of self-prepared isotonic and hypertonic nasal saline irrigation. Proceeding of the 15th Asian Conference on Clinical Pharmacy; 2015 June 23-26; Bangkok, Thailand. Khonkean: Khon Kean University printing house; 2015. 
18. Schulz KF, Altman DG, Moher D, for the CONSORT Group. CONSORT 2010 Statement: updated guidelines for reporting parallel group randomised trials. Ann Int Med. 2010;152:726-32.

19. Moher D, Hopewell S, Schulz KF, Montori V, Gøtzsche PC, Devereaux PJ, et al. CONSORT 2010 explanation and elaboration: updated guidelines for reporting parallel group randomised trials. BMJ. 2010;340:c869.

20. Bunnag C, Leurmarnkul W, Jareoncharsri P, Ungkanont K, Tunsuriyawong P, Kosrirukvongs P. Development of a health-related quality of life questionnaire for Thai Patients with rhinoconjunctivitis. Asian Pac J Allergy Immunol. 2004;22:69-79.

21. Ellis AK, Soliman M, Steacy L, Boulay ME, Boulet LP, Keith PK, et al. The allergic rhinitis - clinical investigator collaborative (AR-CIC): nasal allergen challenge protocol optimization for studying AR pathophysiology and evaluating novel therapies. Allergy Asthma Clin Immunol. 2015;11(1):16.

22. Garavello W, Romagnoli M, Sordo L, Gaini R, Di Berardino C, Angrisano A. Hypersaline nasal irrigation in children with symptomatic seasonal allergic rhinitis: a randomized study. Pediatr Allergy Immunol. 2003;14: 140-3.

23. Ural A, Oktemaer KT, Kizil Y, Ileri F. Impact of isotonic and hypertonic saline solutions mucociliary activity in various nasal pathologies: clinical study. J Laryngol Otol. 2009;123(5):517-21.
24. Keojampa B, Nguyen M, Ryan M. Effects of buffered saline solution on nasal mucociliary clearance and nasal airway patency. Otolaryngol Head Neck Surg. 2004; 131(5):679-82.

25. Talbot AR, Herr T, Parsons D. Mucociliary clearance and buffered hypertonic saline. Laryngoscope. 1997;107:500-3.

26. Chusakul S, Warathanasin S, Suksang-panya N, Phannaso C, Ruxrungtham $\mathrm{S}$, Snidvongs K, et al. Comparison of buffered and non-buffered nasal saline irrigations in treating allergic rhinitis. Laryngoscope. 2013;123(1):53-6.

27. Reddi BAJ. Why is saline so acidic (and does it really matter?). Int J Med Sci. 2013;10(6):747-50.

28. Barnes ML, Vaidyanathan S, Williamson PA, Lipworth BJ. The minimal clinically important difference in allergic rhinitis. Clin Exp Allergy. 2010; 40(2):242-50.

29. Meltzer EO, Wallace D, Dykewicz M, Shneyer L. Minimal clinically important difference (MCID) in allergic rhinitis: agency for healthcare research and ruality or anchor-based thresholds? J Allergy Clin Immunol Pract. 2016;4(4):682-8. 